\title{
A VLA search for young protostars embedded in dense cores
}

\author{
D. Stamatellos ${ }^{1}$, D. Ward-Thompson ${ }^{1,2}$, A. P. Whitworth ${ }^{1}$, and S. Bontemps ${ }^{2}$ \\ ${ }^{1}$ School of Physics \& Astronomy, Cardiff University, 5 The Parade, Cardiff CF24 3AA, Wales, UK \\ e-mail: D.Stamatellos@astro.cf.ac.uk \\ 2 Observatoire de Bordeaux, BP 89, 2 Rue de l'Observatoire, 33270 Floirac, France
}

Received 12 September 2006 / Accepted 6 November 2006

\begin{abstract}
Aims. Four dense cores, L1582A, L1689A, B133 and B68, classified as prestellar in terms of the absence of detectable NIR emission, are observed at radio wavelengths to investigate whether they nurture very young protostars.

Methods. We perform deep radio continuum observations at $3.6 \mathrm{~cm}$ and $6 \mathrm{~cm}$ using the VLA.

Results. No definite young protostars were discovered in any of the four cores observed. A few radio sources were discovered close to the observed cores, but these are most likely extragalactic sources or YSOs unrelated to the cores observed.

In L1582A we discovered a weak radio source near the centre of the core with radio characteristics and offset from the peak of the submillimeter emission similar to that of the newly discovered protostar in the core L1014, indicating a possible protostellar nature for this source. This needs to be confirmed with near- and/or mid-infrared observations (e.g. with Spitzer). Hence based on the current observations we are unable to confirm unequivocally that L1582A is starless.

In L1689A a possible 4.5- $\sigma$ radio source was discovered at the centre of the core, but needs to be confirmed with future observations. In B133 a weak radio source, possibly a protostar, was discovered at the edge of the core on a local peak of the core submm emission, but no source was detected at the centre of the core. Thus, B133 is probably starless, but may have a protostar at its edge.

In B68 no radio sources were discovered inside or at the edge of the core, and thus B68 is indeed starless.

Four more radio sources with spectral indices characteristic of young protostars were discovered outside the cores but within the extended clouds in which these cores reside.

Conclusions. We conclude that the number of cores misclassified as prestellar is probably very small and does not significantly alter the estimated lifetime of the prestellar phase.
\end{abstract}

Key words. stars: formation - radio continuum: stars - ISM: clouds

\section{Introduction}

The identification of the youngest protostars is important for understanding how dense cores in molecular clouds collapse and form stars. The initial conditions for star formation are provided by dense cores in molecular clouds in which there is no evidence that star formation has occurred (starless cores, Myers et al. 1983). Some of these cores are thought to be close to collapse or already collapsing, and they are labelled prestellar cores (e.g. Ward-Thompson et al. 1994; Ward-Thompson et al. 2002). Prestellar cores evolve to Class 0 objects, which represent the youngest protostars (André et al. 1993).

The presence of a protostar in a core is inferred by one or more of the following criteria (André et al. 2000): (i) compact centimeter radio emission; (ii) a bipolar molecular outflow; (iii) NIR or MIR emission. These criteria depend on the sensitivity of the telescope used, and how young and embedded the protostar is in its parent cloud. Additionally, the amount of radio emission from young protostars is uncertain and probably very low (e.g. Curiel et al. 1987; Rodríguez et al. 1989b; Neufeld \& Hollenbach 1996; Harvey et al. 2002; Eiroa et al. 2005). Finally, in many cases the complexity of star forming regions (e.g. dense environment, multiple sources, inflow of material) does not allow a clear identification of all the outflows in a given region. Thus, a null detection with a particular telescope does not necessarily mean that a source does not exist inside a core.
The need for very sensitive observations to discover young protostars residing inside dense cores was demonstrated recently by Young et al. (2004), who, using the Spitzer space telescope, detected a NIR source embedded in L1014 (L1014-IRS), a dense core that was previously classified as starless. This suggests that L1014 is in fact a young Class 0 object. Since the Young et al. discovery, the protostellar nature of L1014-IRS has been confirmed by the detection of a very weak bipolar outflow emanating from the IR source (Bourke et al. 2005) that affects the geometry of the observed scattering light nebula around the source (Huard et al. 2006). Additionally 2 more NIR sources have been discovered inside cores previously thought to be starless: in L1521F in the Taurus molecular cloud (Terebey et al. 2005; Bourke et al. 2006), and in L1148 in the Cepheus flare (Kauffmann et al. 2005). These recently discovered lowluminosity protostars are similar to the previously known Class 0 protostars VLA1623 (André et al. 1993) and HH24MMS (Ward-Thompson et al. 1995; Bontemps et al. 1996). These discoveries of low-luminosity, young protostars, open up the possibility that other cores previously classified as prestellar may in fact contain very young protostars.

Radio observations are suitable for detecting deeply embedded protostars, as long wavelength radiation can escape the large column density of the envelope practically unattenuated. Previous studies (e.g. André et al. 1987; Leous et al. 1991; Bontemps et al. 1995) have identified a large number of young 
protostars exhibiting detectable radio emission. However, very young and low luminosity protostars are believed to produce very weak radio emission (e.g. Harvey et al. 2002; Neufeld \& Hollenbach 1994, 1996), and thus deep observations are needed to detect them.

Young protostars exhibit a positive or almost flat $(>-0.1)$ spectral index (defined as $\alpha$, where $S_{v} \propto v^{a}$ ) between e.g. $3.6 \mathrm{~cm}$ and $6 \mathrm{~cm}$ (André et al. 1987; Anglada et al. 1998; Beltrán et al. 2001). This behaviour of the radio spectral index is characteristic of free-free thermal emission from ionised gas. This is most likely produced by a partially ionised jet that propagates into the collapsing envelope producing shocks (e.g. Curiel et al. 1987). It could also be produced by an ionised disc wind (e.g. Martin 1996) or by the accretion shock that develops on the surface of the protostar, and/or on the surface of the disc that surrounds the protostar, which heats and ionises the infalling gas (Winkler \& Newman 1980; Cassen \& Moosman 1981). However, the latter two mechanisms produce radio emission that is at too low a level to be readily detected.

A negative $(<-0.1)$ radio spectral index indicative of nonthermal gyro-synchrotron emission has also been observed in some T Tauri stars (e.g. André et al. 1988; André 1996; Rodríguez et al. 1999), in a possible Class I object (Feigelson et al. 1998), and even in a possible Class 0 object, the triple radio continuum source in Serpens (Rodríguez et al. 1989a; Curiel et al. 1993; Curiel 1995; Raga et al. 2000). The nonthermal emission of more evolved protostars (Class II, III) is believed to be associated with the magnetic fields around these objects and the star-disc interaction region (e.g. André 1996). The non-thermal emission from younger protostars is thought to be the result of particle acceleration behind a diffuse shock wave (Rodríguez et al. 1989a; Curiel et al. 1993, and references therein).

Stamatellos et al. (2005a,b) performed 3-dimensional radiative transfer calculations on simulations of the collapse of a starforming core and studied the transition from a starless core to a core with an embedded young protostar. These simulations suggest (i) that cores with young protostars appear to be cold ( $T \sim 15-30 \mathrm{~K})$ but still hotter than starless cores $(T<15 \mathrm{~K})$, and (ii) that cores with young protostars appear more circular (at least in their central regions) than starless cores (see also Goodwin et al. 2002). Based on this study, we identified 4 cores classified as starless (L1582A, L1689A, B133, B68) that might in fact contain very young protostars. These cores were selected in terms of their circular appearance and their relatively high estimated temperatures $(\sim 15 \mathrm{~K})$. These cores were observed with the VLA in the continuum at $3.6 \mathrm{~cm}$ and $6 \mathrm{~cm}$.

In this paper we present the results of these radio observations aiming to detect very young embedded sources in dense cores. In Sect. 2 we describe the observational details and the properties of the detected radio sources, in Sect. 3 we summarize our results, and in Sect. 4 we report our conclusion.

\section{Observations and results}

L1582A, L1689A, B133 and B68 were observed at $3.6 \mathrm{~cm}$ using the VLA. The observations were performed in July/August 2005 , in the VLA C configuration. The total integration time was about $\sim 4 \mathrm{~h}$ for each core including time spent on the calibrators. These cores (except B68) were also observed at $6 \mathrm{~cm}$ in October/November 2005, in the DnC configuration. L1582A was observed for $\sim 5 \mathrm{~h}, \mathrm{~B} 133$ for $\sim 5 \mathrm{~h}$, and L1689A for $\sim 1 \mathrm{~h}$. The data were calibrated using the AIPS package of the NRAO.
Table 1. Distances and positions of the dense cores observed.

\begin{tabular}{lccc}
\hline \hline Core & $d^{a}(\mathrm{pc})$ & $\mathrm{RA}^{b}(2000)$ & $\operatorname{Dec}^{b}(2000)$ \\
\hline L1582A & 400 & $05^{\mathrm{h}} 32^{\mathrm{m}} 01.0^{\mathrm{s}}$ & $+12^{\circ} 30^{\prime} 23^{\prime \prime}$ \\
L1689A & 130 & $16^{\mathrm{h}} 32^{\mathrm{m}} 13.2^{\mathrm{s}}$ & $-25^{\circ} 03^{\prime} 45^{\prime \prime}$ \\
B133 & 200 & $19^{\mathrm{h}} 06^{\mathrm{m}} 08.4^{\mathrm{s}}$ & $-06^{\circ} 52^{\prime} 52^{\prime \prime}$ \\
B68 & 130 & $17^{\mathrm{h}} 22^{\mathrm{m}} 39.2^{\mathrm{s}}$ & $-23^{\circ} 50^{\prime} 01^{\prime \prime}$ \\
\hline
\end{tabular}

${ }^{a}$ Distances taken from Kirk et al. (2005); ${ }^{b}$ peak of the $450 \mu \mathrm{m} \&$ $850 \mu \mathrm{m}$ emission (Kirk et al. 2005).

In Table 1 we list the positions of the cores taken from Kirk et al. (2005). These positions correspond to the peak of the $850 \mu \mathrm{m}$ and $450 \mu \mathrm{m}$ emission detected using SCUBA with a beam size $F W H M$ of $14.8^{\prime \prime}$. We use these positions as phase centres. In Table 2 we list the phase calibrators used, their bootstrapped flux densities, the VLA beam size, and the achieved rms noise for each field. Finally, we report the number of radio sources detected and the number of background, extra-galactic sources expected in each field (see below). The amplitude calibrators used were $3 \mathrm{C} 286\left(F_{3.6 \mathrm{~cm}}=5.26 \mathrm{Jy}, F_{6 \mathrm{~cm}}=7.34 \mathrm{Jy}\right)$ and $3 \mathrm{C} 48\left(F_{3.6 \mathrm{~cm}}=3.25 \mathrm{Jy}, F_{6 \mathrm{~cm}}=5.32 \mathrm{Jy}\right)$.

The number of background sources expected in our field is determined using the Anglada et al. (1998) calculation. Assuming that extra-galactic sources have the characteristics described by Condon (1984), the expected number of background sources $\langle N\rangle$ in a field of angular diameter $\theta_{\mathrm{F}}$ is

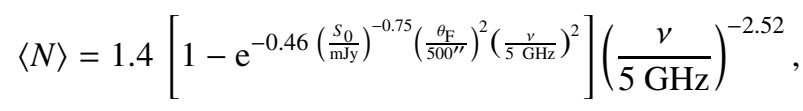

where $S_{0}$ is the minimum detectable flux density at the centre of the field. Each of the fields has an angular diameter of $\theta_{\mathrm{F}} \approx 500^{\prime \prime}$, so the expected number of background sources at $3.6 \mathrm{~cm}$ is

$\langle N\rangle_{3.6 \mathrm{~cm}}=2.6\left(\frac{S_{0}}{50 \mu \mathrm{Jy}}\right)^{-0.75}$,

and at $6 \mathrm{~cm}$,

$\langle N\rangle_{6 \mathrm{~cm}}=3.0\left(\frac{S_{0}}{100 \mu \mathrm{Jy}}\right)^{-0.75}$.

Thus, we expect a total number of $\sim 9 \pm 3$ background sources in all our fields at $3.6 \mathrm{~cm}$, and $\sim 5 \pm 2$ sources at $6 \mathrm{~cm}$. We detected 16 sources at $3.6 \mathrm{~cm}$ and 8 sources at $6 \mathrm{~cm}$, thus the expected number of Galactic sources detected, possibly associated with the star forming regions observed, is $7 \pm 3$.

In Table 3 we list the detected radio sources, their positions, flux densities and peak fluxes, their spectral indices $\alpha$ (in cases where a source has been detected both in $3.6 \mathrm{~cm}$ and $6 \mathrm{~cm}$ ), and their offsets from the peak of the submm emission of the core. The fluxes were corrected for the effect of primary-beam response. We assume a detection limit of 5- $\sigma$ (however we make reference to a possible 4.5- $\sigma$ detection). Most of the sources do not correspond to any sources listed in the SIMBAD astronomical database and are new sources.

In the next subsections we describe in detail the observed radio sources and their characteristics, for each of the cores observed.

\section{1. $L 1582 A$}

In L1582A we discovered a weak radio source (VLA1) close $\left(\sim 18^{\prime \prime}\right)$ to the peak of the submillimeter core emission. 
Table 2. Phase calibrators, beam sizes, rms noise, and number of radio sources detected.

\begin{tabular}{|c|c|c|c|c|c|c|c|c|c|}
\hline \multirow[t]{2}{*}{ Core } & \multicolumn{3}{|c|}{ Phase calibrator(s) } & \multicolumn{2}{|c|}{ Beam (") } & \multicolumn{2}{|c|}{ rms noise $(\mu \mathrm{Jy} / \mathrm{bm})^{a}$} & \multicolumn{2}{|c|}{ Radio sources $^{b}$} \\
\hline & Calibrator & $S_{3.6 \mathrm{~cm}}(\mathrm{Jy})$ & $S_{6 \mathrm{~cm}}(\mathrm{Jy})$ & $3.6 \mathrm{~cm}$ & $6 \mathrm{~cm}$ & $3.6 \mathrm{~cm}$ & $6 \mathrm{~cm}$ & $3.6 \mathrm{~cm}$ & $6 \mathrm{~cm}$ \\
\hline L1582A & $0530+135$ & $2.905 \pm 0.002$ & $3.08 \pm 0.09$ & $3.3 \times 2.7$ & $12.4 \times 6.6$ & 13 & 29 & $4(2.1)$ & $2(2.7)$ \\
\hline L1689A & $\begin{array}{l}1522-275 \\
1626-298\end{array}$ & $\begin{array}{l}1.210 \pm 0.006 \\
2.602 \pm 0.015\end{array}$ & $\begin{array}{c}1.06 \pm 0.07 \\
-\end{array}$ & $5.5 \times 2.9$ & $17.1 \times 10.4$ & 12 & 60 & $6(2.3)$ & $5(1.3)$ \\
\hline B133 & $\begin{array}{l}1939-100 \\
1939-154\end{array}$ & $\begin{array}{c}0.566 \pm 0.002 \\
-\end{array}$ & $\begin{array}{c}- \\
0.55 \pm 0.01\end{array}$ & $3.7 \times 3.0$ & $16.9 \times 9.6$ & 11 & 60 & $4(2.3)$ & $2(1.3)$ \\
\hline B68 & $\begin{array}{l}1743-309 \\
1751-253\end{array}$ & $\begin{array}{l}0.233 \pm 0.001 \\
0.259 \pm 0.001\end{array}$ & $\begin{array}{l}- \\
-\end{array}$ & $5.3 \times 3.0$ & - & 12 & - & $4(2.3)$ & - \\
\hline
\end{tabular}

${ }^{a}$ Mean rms noise in the field in $\mu \mathrm{Jy} /$ beam; ${ }^{b}$ number of radio sources detected in each field. In the parentheses is the expected number of background sources calculated using Eqs. (2), (3).

Table 3. Radio sources detected at 3.6 and $/$ or $6 \mathrm{~cm}$.

\begin{tabular}{|c|c|c|c|c|c|c|c|c|}
\hline \multirow[t]{2}{*}{ Source } & \multicolumn{2}{|c|}{ Position $^{a}$} & \multirow{2}{*}{$\begin{array}{c}S_{3.6 \mathrm{~cm}^{b}} \\
(\mu \mathrm{Jy})\end{array}$} & \multirow{2}{*}{$\begin{array}{c}S_{3.6 \mathrm{~cm}}^{\text {peak }}{ }^{2} \\
(\mu \mathrm{Jy} / \text { beam })\end{array}$} & \multirow{2}{*}{$\begin{array}{l}S_{6 \mathrm{~cm}} \\
(\mu \mathrm{Jy})\end{array}$} & \multirow{2}{*}{$\begin{array}{c}S_{6 \mathrm{~cm}}^{\text {peak }} \\
(\mu \mathrm{Jy} / \text { beam })\end{array}$} & \multirow[t]{2}{*}{$\alpha^{d}$} & \multirow{2}{*}{$\begin{array}{l}\text { Offset }^{e} \\
\quad\left({ }^{\prime \prime}\right)\end{array}$} \\
\hline & RA (2000) & Dec (2000) & & & & & & \\
\hline \multicolumn{9}{|l|}{ L1582A } \\
\hline $1 \ldots \ldots$ & $05^{\mathrm{h}} 32^{\mathrm{m}} 00.5^{\mathrm{s}}$ & $+12^{\circ} 30^{\prime} 40^{\prime \prime}$ & $142 \pm 23$ & $139 \pm 13$ & $280 \pm 530$ & $280 \pm 30$ & $-1.3 \pm 0.5$ & 18 \\
\hline 2 . & $05^{\mathrm{h}} 32^{\mathrm{m}} 06.3^{\mathrm{s}}$ & $+12^{\circ} 32^{\prime} 41^{\prime \prime}$ & $200 \pm 60$ & $137 \pm 26$ & - & - & - & 165 \\
\hline & $05^{\mathrm{h}} 32^{\mathrm{m}} 08.9^{\mathrm{s}}$ & $+12^{\circ} 29^{\prime} 34^{\prime \prime}$ & - & - & $300 \pm 100$ & $180 \pm 40$ & - & 138 \\
\hline \multicolumn{9}{|l|}{ L1689A } \\
\hline $1 \ldots \ldots$ & $16^{\mathrm{h}} 32^{\mathrm{m}} 23.3^{\mathrm{s}}$ & $-25^{\circ} 00^{\prime} 34^{\prime \prime}$ & $8610 \pm 130$ & $8180 \pm 70$ & $5800 \pm 600$ & $4600 \pm 500$ & $0.8 \pm 0.2$ & 235 \\
\hline $2 \ldots \ldots$ & $16^{\mathrm{h}} 32^{\mathrm{m}} 25.4^{\mathrm{s}}$ & $-25^{\circ} 02^{\prime} 16^{\prime \prime}$ & $830 \pm 70$ & $490 \pm 30$ & $1100 \pm 400$ & $640 \pm 170$ & $-0.6 \pm 0.7$ & 188 \\
\hline $3 \ldots \ldots$ & $16^{\mathrm{h}} 32^{\mathrm{m}} 13.9^{\mathrm{s}}$ & $-25^{\circ} 02^{\prime} 25^{\prime \prime}$ & $544 \pm 26$ & $496 \pm 14$ & $750 \pm 70$ & $740 \pm 70$ & $-0.63 \pm 0.21$ & 80 \\
\hline $4 \ldots \ldots$ & $16^{\mathrm{h}} 32^{\mathrm{m}} 14.2^{\mathrm{s}}$ & $-25^{\circ} 02^{\prime} 04^{\prime \prime}$ & $520 \pm 30$ & $347 \pm 15$ & $550 \pm 70$ & $550 \pm 70$ & $-0.11 \pm 0.28$ & 101 \\
\hline $5 \ldots \ldots$ & $16^{\mathrm{h}} 32^{\mathrm{m}} 13.8^{\mathrm{s}}$ & $-25^{\circ} 02^{\prime} 38^{\prime \prime}$ & $440 \pm 40$ & $174 \pm 13$ & $660 \pm 60$ & $640 \pm 60$ & $-0.79 \pm 0.25$ & 67 \\
\hline $6 \ldots \ldots$ & $16^{\mathrm{h}} 32^{\mathrm{m}} 13.3^{\mathrm{s}}$ & $-25^{\circ} 03^{\prime} 45^{\prime \prime}$ & $58 \pm 12$ & $58 \pm 12$ & - & - & - & 1 \\
\hline \multicolumn{9}{|l|}{ B133 } \\
\hline $1 \ldots \ldots$ & $19^{\mathrm{h}} 06^{\mathrm{m}} 05.7^{\mathrm{s}}$ & $-06^{\circ} 50^{\prime} 06^{\prime \prime}$ & $31850 \pm 50$ & $28730 \pm 30$ & $67800 \pm 300$ & $66550 \pm 150$ & $-1.48 \pm 0.01$ & 171 \\
\hline $2 \ldots \ldots$ & $19^{\mathrm{h}} 06^{\mathrm{m}} 14.0^{\mathrm{s}}$ & $-06^{\circ} 49^{\prime} 35^{\prime \prime}$ & $730 \pm 130$ & $420 \pm 50$ & - & - & $>1.7 \pm 0.3$ & 214 \\
\hline $3 \ldots \ldots$ & $19^{\mathrm{h}} 06^{\mathrm{m}} 08.4^{\mathrm{s}}$ & $-06^{\circ} 53^{\prime} 32^{\prime \prime}$ & $120 \pm 30$ & $71 \pm 12$ & - & - & - & 40 \\
\hline $4 \ldots \ldots$ & $19^{\mathrm{h}} 06^{\mathrm{m}} 07.0^{\mathrm{s}}$ & $-06^{\circ} 53^{\prime} 58^{\prime \prime}$ & $100 \pm 25$ & $81 \pm 13$ & - & - & - & 69 \\
\hline \multicolumn{9}{|l|}{$\mathrm{B} 68^{f}$} \\
\hline $1 \ldots \ldots$ & $17^{\mathrm{h}} 22^{\mathrm{m}} 35.0^{\mathrm{s}}$ & $-23^{\circ} 47^{\prime} 58^{\prime \prime}$ & $9310 \pm 40$ & $8550 \pm 21$ & - & - & - & 136 \\
\hline $2 \ldots \ldots$ & $17^{\mathrm{h}} 22^{\mathrm{m}} 31.8^{\mathrm{s}}$ & $-23^{\circ} 48^{\prime} 18^{\prime \prime}$ & $223 \pm 24$ & $223 \pm 24$ & - & - & - & 145 \\
\hline $3 \ldots \ldots$ & $17^{\mathrm{h}} 22^{\mathrm{m}} 28.0^{\mathrm{s}}$ & $-23^{\circ} 50^{\prime} 32^{\prime \prime}$ & $410 \pm 26$ & $410 \pm 26$ & - & - & - & 157 \\
\hline $4 \ldots \ldots$ & $17^{\mathrm{h}} 22^{\mathrm{m}} 35.4^{\mathrm{s}}$ & $-23^{\circ} 52^{\prime} 34^{\prime \prime}$ & $150 \pm 50$ & $143 \pm 27$ & - & - & - & 166 \\
\hline
\end{tabular}

${ }^{a}$ Positional errors are expected to be $\sim 1^{\prime \prime} ;{ }^{b}$ Flux density ( $\mu \mathrm{Jy}$ ) and 1- $\sigma$ errors, corrected for the primary beam response; ${ }^{c}$ peak flux density $\left(\mu \mathrm{Jy} /\right.$ beam) and 1- $\sigma$ errors, corrected for the primary beam response; ${ }^{d}$ spectral index between 3.6 and $6 \mathrm{~cm} ;{ }^{e}$ angular displacement of the source from the peak of the submm emission; ${ }^{f} \mathrm{~B} 68$ was not observed at $6 \mathrm{~cm}$.

The source is observed both in $3.6 \mathrm{~cm}$ and $6 \mathrm{~cm}$ (see Figs. 1, 2). The spectral index of the source is $\alpha=-1.2 \pm 0.5$. This spectral index is not what is expected for Class 0 protostars. For example, for the prototypical Class 0 object VLA1623 (André et al. 1993) $\alpha=0.6$. The spectral index of the radio emission from protostars is thought to be between -0.1 and 2 , consistent with thermal free-free emission, whereas for extra-galactic sources it is expected to be $<-0.1$, characteristic of non-thermal emission due to optically thin synchrotron emission (e.g. André et al. 1987; Anglada et al. 1998; Beltran et al. 2001). Thus, L1582A-VLA1 is most probably an extragalactic source.

However we note that recent observations of the confirmed low luminosity protostar L1014-IRS (Shirley et al., VLA proposal) indicate a similar spectral index. The unusual negative spectral index may be due to non-thermal (synchrotron) emission. The similarities of L1582A-VLA1 to L1014-IRS (similar $3.6 \mathrm{~cm}$ and $6 \mathrm{~cm}$ fluxes, similar offsets from the peak of the submillimeter emission of the core) are suggestive that
L1582A-VLA1 may also be a very young protostar embedded in the core, but this cannot be confirmed with the current observations.

Two more sources were detected in the field. VLA2 was detected only at $3.6 \mathrm{~cm}$ but a negative spectral index cannot be excluded. This source is outside the core but within the extended CO $(J=1-0)$ line emission (Dame et al. 2001; see also Kirk et al. 2005). VLA3 was detected only at $6 \mathrm{~cm}$ and thus has a negative spectral index and should be a background source.

Thus, we are unable to confirm unequivocally that L1582A is starless.

\section{2. $L 1689 A$}

In the L1689A field we discovered 6 sources, 5 of which were detected both at $3.6 \mathrm{~cm}$ and $6 \mathrm{~cm}$, and 1 marginally detected only at $3.6 \mathrm{~cm}$. 


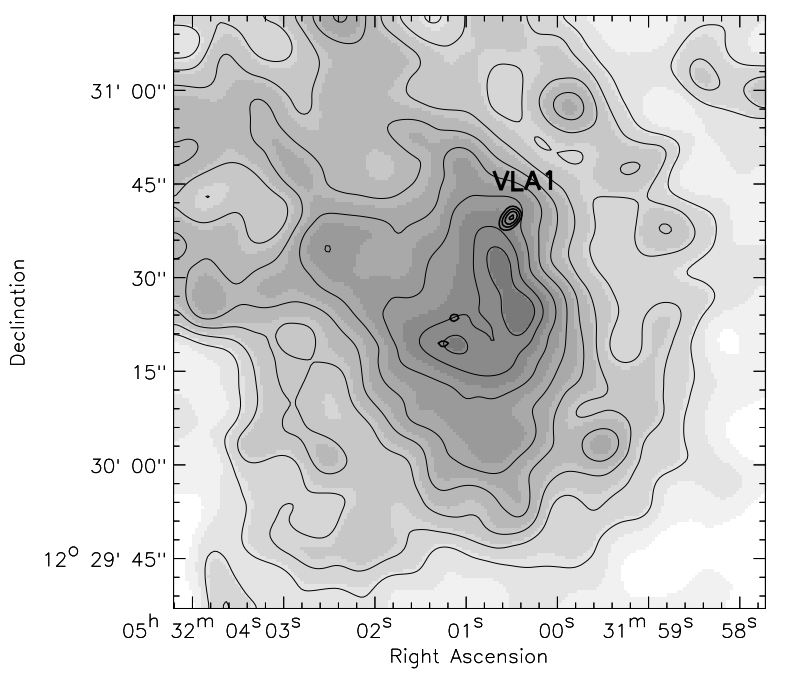

Fig. 1. L1582A: $3.6 \mathrm{~cm}$ VLA observations (solid thick contours) overlaid on $850 \mu \mathrm{m}$ SCUBA observations from Kirk et al. (2005) (grayscale and solid thin contours). $3.6 \mathrm{~cm}$ solid contours are plotted at $52 \mu \mathrm{Jy}$ $(5-\sigma) \times(1,1.5,2,2.5,3) .850 \mathrm{~mm}$ dashed contours are plotted at 0.3 , $0.4,0.5,0.6,0.7,0.8,0.9$ and 0.95 of the maximum $850 \mathrm{~mm}$ flux $(170 \mathrm{mJy} /$ beam $)$. The VLA beam size is mentioned in Table 2 . The SCUBA beam size at $850 \mu \mathrm{m}$ is $14^{\prime \prime}$. A radio source has been discovered near the centre of the core.

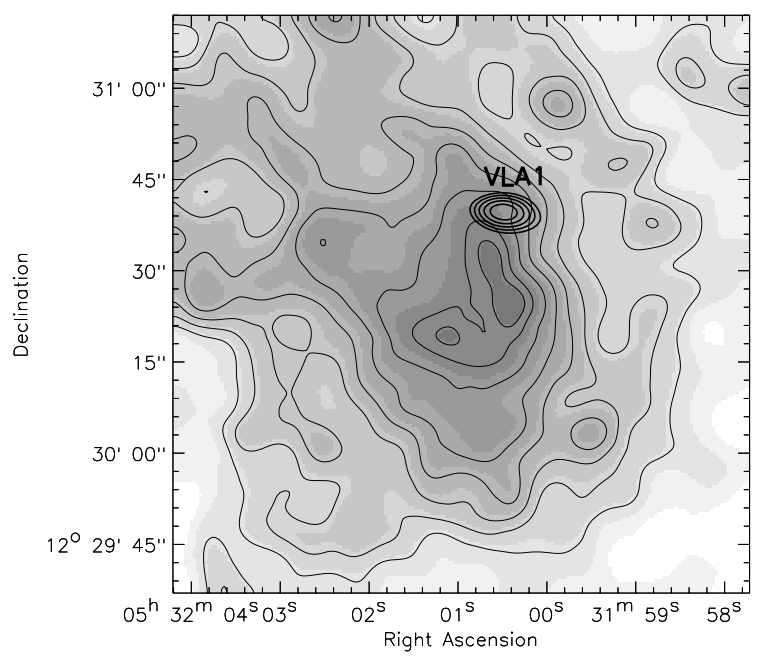

Fig. 2. L1582A: $6 \mathrm{~cm}$ VLA observations (solid thick contours) overlaid on $850 \mu \mathrm{m}$ SCUBA observations from Kirk et al. (2005) (grayscale and solid thin contours). $6 \mathrm{~cm}$ solid contours are plotted at $145 \mu \mathrm{Jy}$ $(5-\sigma) \times(1,1.2,1.4,1.6,1.8) .850 \mathrm{~mm}$ dashed contours are plotted as in Fig. 1. The VLA beam size is mentioned in Table 2. The SCUBA beam size at $850 \mu \mathrm{m}$ is $14^{\prime \prime}$.

VLA1 has a positive spectral index, indicative of free-free thermal emission from ionised gas. It is outside the main core but within the extended CO line emission of the Ophiuchus GMC (Dame et al. 2001; Kirk et al. 2005). It coincides with a local column density peak of the cloud (see Fig. 3; and Nutter et al. 2006), although the dust emission is rather weak.

VLA2 is also outside the core but within the extended $\mathrm{CO}$ emission. The sign of its spectral index cannot be determined; it could be either a young star or a background source.

Sources VLA3, VLA4 and VLA5 were detected in both wavelengths and they seem to be related, as they are aligned. They are located outside the L1689A core but they are coincident with a submm filament adjacent to the core. The total

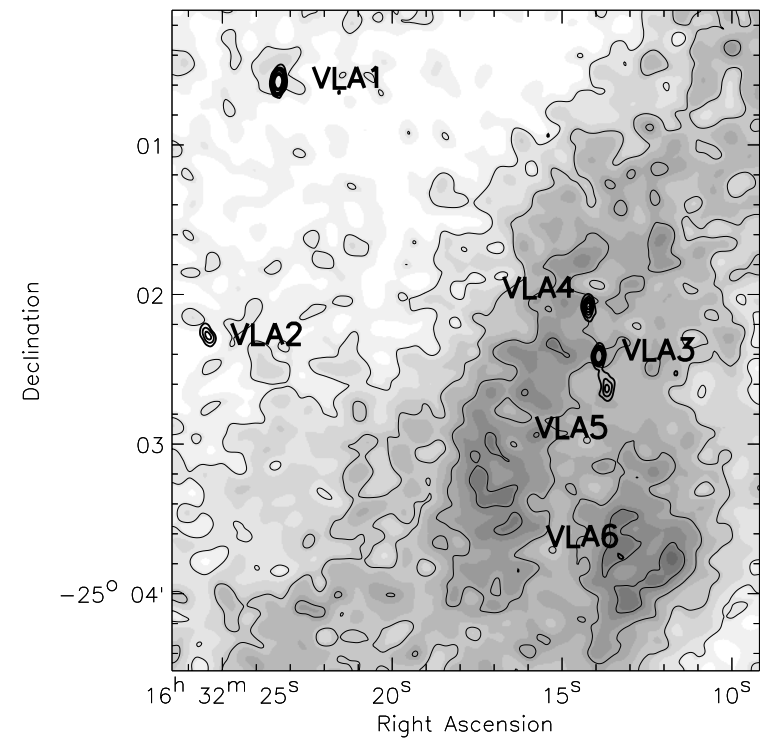

Fig. 3. L1689A: $3.6 \mathrm{~cm}$ VLA observations (solid thick contours) overlaid on $850 \mu \mathrm{m}$ SCUBA observations from Nutter et al. (2006) (grayscale and thin solid contours). $3.6 \mathrm{~cm}$ solid contours are plotted at $54 \mu \mathrm{Jy}(4.5-\sigma) \times(1,2,4,6,8,10,12) .850 \mathrm{~mm}$ dashed contours are plotted at $0.2,0.4,0.6$, and 0.8 of the maximum $850 \mathrm{~mm}$ flux (170 mJy/beam).The VLA beam size is mentioned in Table 2. The SCUBA beam size at $850 \mu \mathrm{m}$ is $14^{\prime \prime}$.

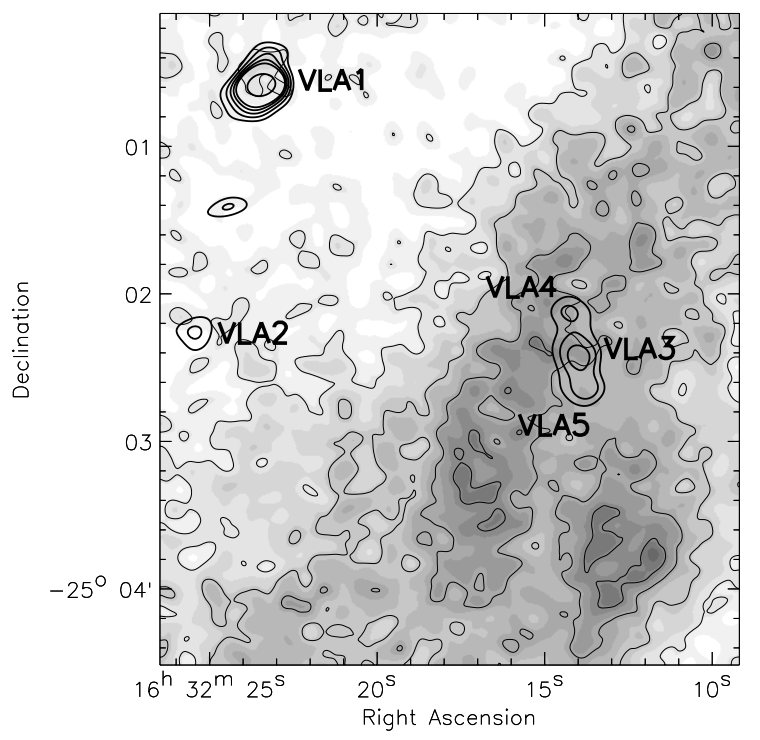

Fig. 4. L1689A: $6 \mathrm{~cm}$ VLA observations (solid thick contours) overlaid on $850 \mu \mathrm{m}$ SCUBA observations from Nutter et al. (2006) (grayscale and thin solid contours). $6 \mathrm{~cm}$ solid contours are plotted at $300 \mu \mathrm{Jy}$ $(5-\sigma) \times(1,1.5,2,2.5,3,6,9) .850 \mathrm{~mm}$ dashed contours are plotted as in Fig. 3. The VLA beam size is mentioned in Table 2. The SCUBA beam size at $850 \mu \mathrm{m}$ is $14^{\prime \prime}$.

projected extent of the structure is $\sim 46^{\prime \prime}$ (i.e. $\sim 6000 \mathrm{AU}$ if they are physically located close to L1689A). The central (VLA3) and the southern (VLA5) source have negative spectral indices (characteristic of non-thermal synchrotron emission), whereas the northern source (VLA4) may have positive or marginally negative spectral index (characteristic of optically thin thermal emission). Thus, these sources are probably extragalactic.

The last source in this field, VLA6, was marginally detected at $3.6 \mathrm{~cm}$ (4.5- $\sigma$ detection). It is located at the centre of the core. It was not detected at $6 \mathrm{~cm}$ but a negative spectral index cannot 


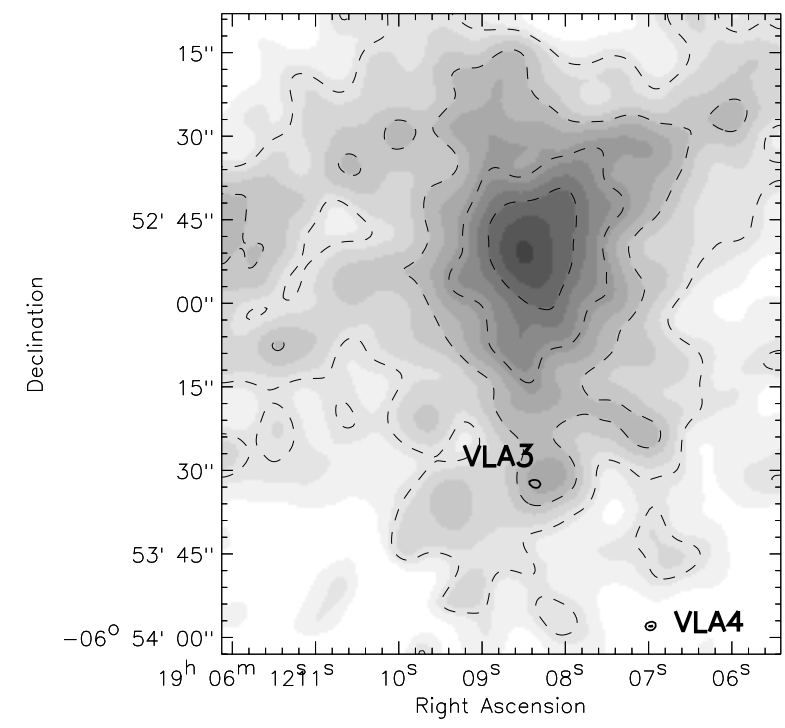

Fig. 5. B133: $3.6 \mathrm{~cm}$ VLA observations (solid contours) overlaid on $850 \mu \mathrm{m}$ SCUBA observations from Kirk et al. (2006) (grayscale and dashed contours). $3.6 \mathrm{~cm}$ solid contours are plotted at $60 \mu \mathrm{Jy}(5-\sigma) \times$ $(1,1.1,1.2,1.3) .850 \mathrm{~mm}$ dashed contours are plotted at $0.2,0.4,0.6$, and 0.8 of the maximum $850 \mathrm{~mm}$ flux ( $170 \mathrm{mJy} /$ beam $)$. The VLA beam size is mentioned in Table 2. The SCUBA beam size is $14^{\prime \prime}$.

be ruled out. The presence of this source in the centre of the core, if indeed it is real, would explain the temperature increase toward the centre of the core suggested by the observations of Ward-Thompson et al. (2002). Thus, there is the possibility that L1689A may not be starless.

\section{3. $B 133$}

In the B133 field we discovered 4 radio sources. The strongest one, B133-VLA1, is located outside the core. It has a negative spectral index between 3.6 and $6 \mathrm{~cm}$, indicating non-thermal emission due to optically thin synchrotron emission characteristic from an extra-galactic source. This source was the only one from this field that was detected at $6 \mathrm{~cm}$. A search in the SIMBAD database reveals a match with PMNJ1906-0650 of the Parkes-MIT-NRAO (PMN) Surveys (Griffith et al. 1995) at $4.85 \mathrm{GHz}\left(S_{v}=65 \pm 11 \mathrm{mJy}\right)$. The source has been detected by the NRAO VLA Sky Survey (Condon et al. 1998) at $1.4 \mathrm{GHz}\left(S_{v}=295.5 \pm 8.9 \mathrm{mJy}\right)$, and by the Texas Survey of Discrete Radio Sources (Douglas et al. 1996) at $365 \mathrm{MHz}$ $\left(S_{v}=1216 \pm 41 \mathrm{mJy}\right)$. These fluxes are also consistent with thin synchrotron emission from an extra-galactic source.

The second strongest source in the field, B133-VLA2, is located outside the core and it is not associated with any column density peak. The absence of detectable $6 \mathrm{~cm}$ emission at the $300 \mu \mathrm{Jy}$ level indicates a positive spectral index $(\alpha>1.7 \pm 0.2)$. This suggests free-free thermal emission. B133-VLA2 may be associated with the Aquila Rift, where B133 is thought to reside (Kirk et al. 2005).

The B133-VLA3 source is located at the edge of the core within a local column density peak. It has been marginally detected (at the $\sim 6-\sigma$ level) and it could be a very young protostar. Unfortunately, although it is not detected at $6 \mathrm{~cm}$ a negative spectral index cannot be excluded, so it may also be an extragalactic source.

Finally, B133-VLA4 is marginally detected at $3.6 \mathrm{~cm}$. It is not detected at $6 \mathrm{~cm}$ but a negative spectral index is possible.
Thus, based on the VLA observations B133 appears to be a starless core with a possible protostar (B133-VLA3) at its edge.

\section{4. $B 68$}

Four radio sources were discovered in the B68 field at $3.6 \mathrm{~cm}$. All of them are outside the core and they are not associated with the core but they are within the extended CO emission (Dame et al. 2001; Kirk et al. 2005) around the core. This core was not observed at $6 \mathrm{~cm}$, and thus the spectral indices of these sources and their nature cannot be determined. We conclude that B68 is starless.

\section{Summary}

We conducted continuum VLA radio observations of 4 dense cores previously classified as prestellar, searching for radio emission from deeply embedded protostars. 17 radio sources were detected in the observed fields, i.e. more than the expected number of background sources. Thus at least a few of them may be associated with the star forming regions observed.

Two radio sources were discovered near the centres of the cores. L1582A-VLA1 is located within $18^{\prime \prime}$ of the peak of the submm emission of the core, and its similarity with the source embedded in L1014 suggests that this may also be a very young protostar embedded in the core, despite its negative spectral index between 3.6 and $6 \mathrm{~cm}$, and its offset from the centre of the core. L1689A-VLA6 is located within $1^{\prime \prime}$ of the peak of the submm emission, and it may also be a very young protostar embedded in the core. However, this detection was only at the 4.5- $\sigma$ level. Future Spitzer data should confirm whether these are young protostars or not. If these two sources indeed are associated with the observed cores then they are similar to the recently discovered low-luminosity protostars, such as that in L1521F, which are young Class 0 objects, e.g. like VLA1623 (André et al. 1993) and HH24MMS (Ward-Thompson et al. 1995; Bontemps et al. 1996). However, based on the current observations and the locations of the radio sources, we conclude that L1582A and L1689A are most likely starless.

Two radio sources were discovered close to edges of cores. L1689A-VLA345, at the edge of the L1689A, is probably an extragalactic source. B133-VLA3, at the edge of B133, coincides with a local peak in the core submillimeter emission, and it may be a young protostar.

Four radio sources with positive spectral indices were detected outside the cores (L1689A-VLA1, L1689A-VLA2, B133-VLA2, B133-VLA4) and, thus could also be young protostars or stars associated with the extended star forming regions where these cores reside.

All of the candidate protostars reported are expected to be detectable in the NIR by the Spitzer space telescope and such observations are needed to confirm whether these sources are indeed protostars. Additionally, sensitive molecular line radio observations, with e.g. the Submillimeter Array (SMA) should be able to detect molecular outflows emanating from these objects, if they are protostars.

\section{Conclusion}

In this paper we presented deep radio observations of 4 prestellar cores, which are relatively warm and almost circular (in their inner regions). According to the Stamatellos et al. (2005) criteria these cores are, among cores presently classified as prestellar, the 
ones most likely to nurture very young protostars. However, we find no definite protostars at the centres of these cores. Two radio sources that could be very young embedded protostars were discovered near the centres of L1582A and L1689A, but further near- or mid-infrared observations (e.g. with Spitzer or the upcoming Herschel) are needed for safe conclusions on the nature of these sources. Hence, based on the current observations, similarly to Kirk et al. (2006) we conclude that the number of cores misclassified as prestellar is probably very small and does not affect significantly the estimated lifetime of the prestellar phase.

Acknowledgements. We thank C. Chandler for her help in reducing and calibrating the VLA data at NRAO Sorocco, and J. Kirk and D. Nutter for providing the SCUBA data. The James Clerk Maxwell Telescope is operated by The Joint Astronomy Centre on behalf of the Particle Physics and Astronomy Research Council of the United Kingdom, the Netherlands Organisation for Scientific Research, and the National Research Council of Canada. The JCMT data shown in this paper were taken during observing runs M96BU56, M97BU88, M99AU34 and M00AU32. This work was carried out while DWT was on sabbatical at l'Observatoire de Bordeaux and CEA, Saclay, and he wishes to thank both institutions for the hospitality accorded to him. We also acknowledge support by PPARC grant PPA/G/O/2002/00497.

\section{References}

André, P. 1996, Radio Emission from the Stars and the Sun, ASP Conf. Ser., 93, 273

André, P., Montmerle, T., \& Feigelson, E. D. 1987, AJ, 93, 1182

André, P., Montmerle, T., Feigelson, E. D., Stine, P. C., \& Klein, K.-L. 1988, ApJ, 335, 940

André, P., Ward-Thompson, D., \& Barsony, M. 1993, ApJ, 406, 122

André, P., Ward-Thompson, D., \& Barsony, M. 2000, Protostars and Planets IV, 59

Anglada, G., Villuendas, E., Estalella, R., et al. 1998, AJ, 116, 2953

Beltrán, M. T., Estalella, R., Anglada, G., Rodríguez, L. F., \& Torrelles, J. M. 2001, AJ, 121, 1556

Bontemps, S., André, P., \& Ward-Thompson, D. 1995, A\&A, 297, 98

Bontemps, S., Ward-Thompson, D., \& Andre, P. 1996, A\&A, 314, 477

Bourke, T. L., Crapsi, A., Myers, P. C., et al. 2005, ApJ, 633, L129

Bourke, T. L., Myers, P. C., Evans, N. J., II, et al. 2006, ApJ, 649, L37

Cassen, P., \& Moosman, A. 1981, Icarus, 48, 353

Condon, J. J. 1984, ApJ, 287, 461
Condon, J. J., Cotton, W. D., Greisen, E. W., et al. 1998, AJ, 115, 1693

Curiel, S. 1995, Rev. Mex. Astron. Astrofis. Conf. Ser., 1, 59

Curiel, S., Canto, J., \& Rodríguez, L. F. 1987, Rev. Mex. Astron. Astrofis., 14, 595

Curiel, S., Rodríguez, L. F., Moran, J. M., \& Canto, J. 1993, ApJ, 415, 191

Dame, T. M., Hartmann, D., \& Thaddeus, P. 2001, ApJ, 547, 792

Douglas, J. N., Bash, F. N., Bozyan, F. A., Torrence, G. W., \& Wolfe, C. 1996, AJ, 111,1945

Eiroa, C., Torrelles, J. M., Curiel, S., \& Djupvik, A. A. 2005, AJ, 130, 643

Feigelson, E. D., Carkner, L., \& Wilking, B. A. 1998, ApJ, 494, L215

Griffith, M. R., Wright, A. E., Burke, B. F., \& Ekers, R. D. 1995, ApJS, 97, 347

Goodwin, S. P., Ward-Thompson, D., \& Whitworth, A. P. 2002, MNRAS, 330, 769

Harvey, D. W. A., Wilner, D. J., Di Francesco, J., et al. 2002, AJ, 123, 3325

Huard, T. L., Myers, P. C., Murphy, D. C., et al. 2006, ApJ, 640, 391

Kauffmann, J., Bertoldi, F., Evans, N. J., \& the C2D Collaboration 2005, Astron. Nachr., 326, 878

Kirk, J. M., Ward-Thompson, D., \& André, P. 2005, MNRAS, 360, 1506

Kirk, J. M., Ward-Thompson, D., \& André, P. 2006, [arXiv:astro-ph/0611165]

Leous, J. A., Feigelson, E. D., André, P., \& Montmerle, T. 1991, ApJ, 379, 683

Martin, S. C. 1996, ApJ, 473, 1051

Myers, P. C., Linke, R. A., \& Benson, P. J. 1983, ApJ, 264, 517

Neufeld, D. A., \& Hollenbach, D. J. 1994, ApJ, 428, 170

Neufeld, D. A., \& Hollenbach, D. J. 1996, ApJ, 471, L45

Nutter, D., Ward-Thompson, D., \& André, P. 2006, MNRAS, 368, 1833

Raga, A. C., Curiel, S., Rodríguez, L. F., \& Cantó, J. 2000, A\&A, 364, 763

Rodríguez, L. F., Curiel, S., Moran, J. M., et al. 1989a, ApJ, 346, L85

Rodríguez, L. F., Myers, P. C., Cruz-Gonzalez, I., \& Terebey, S. 1989b, ApJ, 347,461

Rodríguez, L. F., Anglada, G., \& Curiel, S. 1999, ApJS, 125, 427

Stamatellos, D., Whitworth, A. P., Boyd, D. F. A., \& Goodwin, S. P. 2005a, A\&A, 439, 159

Stamatellos, D., Whitworth, A. P., \& Goodwin, S. P. 2005b, Astron. Nachr., 326, 882

Terebey, S., van Buren, D., Brundage, M. \& Hancock, T. 2005, Protostars and Planets V, Proc. Conference held October 24-28, 2005, in Hilton Waikoloa Village, Hawai'i. LPI Contribution No. 1286., 8611

Ward-Thompson, D., Scott, P. F., Hills, R. E., \& André, P. 1994, MNRAS, 268, 276

Ward-Thompson, D., Chini, R., Krugel, E., Andre, P., \& Bontemps, S. 1995, MNRAS, 274, 1219

Ward-Thompson, D., André, P., \& Kirk, J. M. 2002, MNRAS, 329, 257

Winkler, K.-H. A., \& Newman, M. J. 1980, ApJ, 236, 201

Young, C. H., Jørgensen, J. K., Shirley, Y. L., et al. 2004, ApJS, 154, 396 